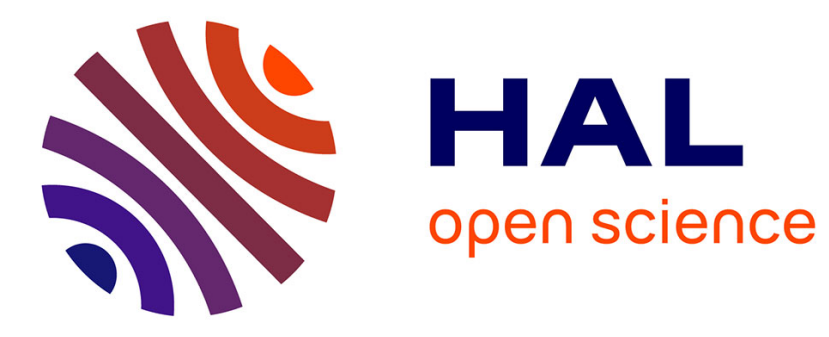

\title{
Interpretability of fuzzy linguistic summaries
}

Marie-Jeanne Lesot, Gilles Moyse, Bernadette Bouchon-Meunier

\section{To cite this version:}

Marie-Jeanne Lesot, Gilles Moyse, Bernadette Bouchon-Meunier. Interpretability of fuzzy linguistic summaries. Fuzzy Sets and Systems, 2016, 292, pp.307-317. 10.1016/j.fss.2014.10.019 . hal01097029

\section{HAL Id: hal-01097029 \\ https://hal.science/hal-01097029}

Submitted on 8 Jan 2015

HAL is a multi-disciplinary open access archive for the deposit and dissemination of scientific research documents, whether they are published or not. The documents may come from teaching and research institutions in France or abroad, or from public or private research centers.
L'archive ouverte pluridisciplinaire HAL, est destinée au dépôt et à la diffusion de documents scientifiques de niveau recherche, publiés ou non, émanant des établissements d'enseignement et de recherche français ou étrangers, des laboratoires publics ou privés. 


\title{
Interpretability of Fuzzy Linguistic Summaries
}

\author{
Marie-Jeanne Lesot ${ }^{\mathrm{a}, \mathrm{b}}$, Gilles Moyse ${ }^{\mathrm{a}, \mathrm{b}, *}$, Bernadette Bouchon-Meunier ${ }^{\mathrm{a}, \mathrm{b}}$ \\ ${ }^{a}$ Sorbonne Universités, UPMC Univ Paris 06, UMR 7606, LIP6, F-75005, Paris, France \\ ${ }^{b}$ CNRS, UMR 7606, LIP6, F-75005, Paris, France
}

\begin{abstract}
This paper investigates the question of the interpretability of fuzzy linguistic summaries, both at the sentence level and at the summary level, seen as a set of sentences. The individual sentence interpretability is examined as depending both on its representativity measured by a quality degree and on its linguistic expression. Different properties at the summary level are also discussed, namely their consistency, their non redundancy and the information they convey.
\end{abstract}

Keywords: Fuzzy linguistic summaries, Interpretability, Summary consistency, Generalised protoform

\section{Introduction}

The interpretability of information handled automatically by machines has always been a difficult problem. For instance, the attempts of humans to extract knowledge from large amounts of data or to interpret the functioning of complex systems motivated researches in domains such as association rules in databases or expert systems, to name a few. The increasing size of available data in the digital world and the diversity of medias and kinds of data, be they images, time series or databases, for instance, put pressure on researchers to provide efficient methods to mine and summarise information in an easily understandable form, to help users such as decision makers or medical doctors to manage efficiently complex cases.

Fuzzy set-based methods are certainly useful in such perspectives for their capacity to process linguistic information through the interface they provide between numerical and symbolic values, and also for their intrinsic ability to reduce complexity by providing a synthesis of individual elements. They enable users to have more friendly interactions with machines than many other methods of computational intelligence. Their interpretability has notwithstanding been strongly questioned and methods to improve it have been proposed.

Interpretability of fuzzy models is a very complex criterion, difficult to define precisely, partly subjective and depending on the context of utilisation. It has been extensively studied in the

\footnotetext{
This work is dedicated to Francesc Esteva, for his pioneering work in fuzzy logic and his dedication to the fuzzy community, especially in Europe.

* Corresponding author

Email address: surname.name@lip6.fr (Gilles Moyse)
} 
case of fuzzy rule-based systems [1, 2, 3, 4], which have been the most widespread within all fuzzy modelling methods, mainly because of the early success of fuzzy expert systems and fuzzy control, both based on rules of the form "if $V_{1}$ is $A_{1}$ and $V_{2}$ is $A_{2}, \ldots$, then $W$ is $B$ ". These studies point out the difficulty to assess interpretability and provide various kinds of properties involved in its evaluation. Compactness, completeness, consistency on the one hand, coverage, normality and distinguishability of fuzzy modalities on the other hand, are considered as the most relevant properties interesting to maximise when looking for a trade-off between expressibility of fuzzy rule-based systems and accuracy of the model.

A growing interest has been expressed more recently for fuzzy linguistic summaries that provide a textual description of numerical data. They have been introduced decades ago and are more and more studied because of the nowadays difficulty to grasp efficiently all available digital information. Textual representation of information can be more efficient than graphical ones in several cases. For instance, the data can be described in high dimensional domains, possibly and generally speaking hard to show graphically, in which case the linguistic summary is an interesting alternative [5]. Moreover, it has been shown that information displayed as text to the user is interpreted more swiftly compared to graphs [6]. Finally, a linguistic summary can be read out by a text-to-speech synthesis system when the visual attention must not be disturbed, while executing a complex task for instance [7], or when it is deficient.

As remarked in [8], the interpretability of fuzzy linguistic summaries has not been much studied as compared to fuzzy rule-based systems, probably for two reasons. First of all, less attention has been brought to them than fuzzy rule-based though they have been introduced in 1982 [9] and mainly investigated since 2001 [10]. Secondly, there exist various kinds of linguistic summaries going beyond the protoforms originally proposed and capturing the generality of the concept is not an easy task.

We can distinguish two points of view to analyse the interpretability of linguistic summaries. The first one considers a process of linguistic summarisation taking into account a list of generalised protoforms based on a number of quantifiers or temporal indications appropriate for the addressed problem, and fuzzy characterisations based on partitions of universes of definition of attributes. In this framework, the above-mentioned properties involved in the interpretability of fuzzy rule-based models are still useful to evaluate with regard to both lists of quantifiers or temporal indications and fuzzy characterisations.

Another point of view, especially relevant in the case of general and more complex forms of fuzzy summaries, takes into account more various elements used to construct them and their interpretability then lies on external considerations corresponding to their acceptability by users or their relevance in terms of natural knowledge.

In this paper, after discussing the general definition of fuzzy linguistic summaries in Section 2, we propose to investigate the question of the interpretability of fuzzy linguistic summaries following a two level approach: first, at the sentence level in Section 3, then at the summary level, seen as a set of sentences in Section 4. 


\section{Fuzzy linguistic summaries}

Fuzzy linguistic summaries can be defined as texts made of several sentences that describe distinct characteristics of a given data set.

The individual sentences that make up the summary are most of the time seen as schemata that are instantiated with respect to the considered data set, a schema being called a protoform [9, 11]. Many variants have been proposed, from " $Q y$ 's are $A$ " and " $Q B y$ 's are $A$ " [9] to more complex ones, as discussed below. Their variety both depends on the type of information they extract from the data and on the type of data they apply to, e.g. data described by numerical attributes or times series.

We propose to consider generalised protoforms as " $M B y$ 's are $A$ " where $M$ is a mode, that describes the extent to which " $B y$ 's are $A$ " holds for the considered data, adjusting its meaning. $M$ can be either a quantifier, as in classic protoforms $[9,11]$, or a temporal indication in the case where the data are time series, as discussed below. $A$ and $B$ are fuzzy modalities, respectively called summariser and qualifier, of linguistic variables used to describe the data; $y$ denotes elements of the dataset to be summarised.

Classic protoforms. The notion of protoforms [11] using quantifiers [12] applied to summaries [9] has been introduced in the case of numerical attribute data, as " $Q y$ 's are $A$ " or more generally " $Q B y$ 's are $A$ ". They can be illustrated by sentences such as "most employees are moderately paid" or "most of young employees are moderately paid".

The mode $M$ is then a fuzzy quantifier [13] that gives information about the quantity of data to which " $B y$ 's are $A$ " applies: it extends the classic $\forall$ and $\exists$ logical quantifiers and can be either absolute or relative. Examples of absolute fuzzy quantifiers include expressions such as "about 10 " or "more than 50", examples of relative fuzzy quantifiers are "less than $10 \%$ " or "most".

Protoforms for time series. Linguistic summaries have been applied to other types of data, in particular time series, i.e. data whose description includes a time attribute. Their specificity induces different types of extracted information and thus different types of summaries.

A first approach consists in extracting numerical attributes from the time series, for instance regarding trends, i.e. piecewise linear approximations of time series, described e.g. through their slope, duration or approximation quality [14]. Such duration-based protoforms lead to summaries such as "most slowly decreasing trends are of a large variability" or "slowly decreasing trends that took most of the time are of a large variability" [14].

A second approach aims at generating summaries considering several time series together. In [15], the protoform " $Q B y$ 's are $A Q_{\tau}$ time" is proposed, illustrated by a sentence such as "few patients have a medium value of heart rate most of the time". In order to evaluate this protoform, the values of each time series for different time stamps are first summarised by the protoform “ $Q_{\tau}$ time $B y$ 's are $A$ ", where $Q_{\tau}$ is a quantifier applied to the time attribute. More precisely, $Q_{\tau}$ is a fuzzy quantifier similar to the ones mentioned above, applied to the time stamps to which the attribute values describing the data are associated. In a second step, the obtained results are summarised across all considered time series, considering a second fuzzy quantifier $Q$.

The evaluation of " $Q_{\tau}$ time $Q B y$ 's are $A$ " [15] is also a two-step process where the set of time series is first summarised at each time stamp and then summarised across all time stamps. 
Another approach dedicated to the comparison of two time series is also proposed, allowing for instance the evaluation of the sentence "most days of year 2001, both series exhibit a local change with the same sign" [16]. In the latter work, in addition, the summary applies to attributes extracted to describe the evolution of the time series and their changes. It must be underlined that such protoforms raise the issue of time scale correlation, as it requires that the time stamps of each series can be aligned.

Temporal protoforms. A distinct type of linguistic summaries for time series takes into account the specificity of the time attribute: it does not apply fuzzy quantifiers but considers the mode $M$ as a temporal indication.

This approach for instance leads to protoforms of the form "regularly $y$ 's are $A$ ", where the adverb "regularly" indeed describes the extent to which " $y$ 's are $A$ " applies considering a specific temporal adjustment $[17,18]$. Such a protoform can be enriched with information about the period, as "Adv every $p$ units, $y$ 's are A", where Adv is an adverb as "roughly" or "exactly", $p$ is an approximation of the period and "unit" a unit considered the most appropriate to express the period, as detailed in Section 3.3.

Fuzzy temporal propositions or FTP [19, 20], that we propose to interpret using the notions of summarisers and quantifiers, encompass several types of temporal indications for time series: examples of obtained summaries include "throughout the last 30 minutes, temperature was high" and "at some instant between 15 minutes and 1 hour after the beginning of the irradiation, temperature was low". More precisely, the summarisers can either characterise the time series values or compare them to that of other time series. The mode $M$ of FTP is defined as a pair of a fuzzy temporal reference, denoted $M_{\tau}$ below, and a persistence mode, linguistically introduced by "in" or "throughout": an absolute $M_{\tau}$ describes a time stamp interval, e.g. "between 1:00pm and 1:30pm" or, in a fuzzy way, "around 08:00am"; a relative $M_{\tau}$ gives a temporal indication in comparison with the current date, e.g. "ten minutes ago" or to a reference event " 10 minutes before $e$ " where $e$ is another characterisation of the data. The persistence mode respectively indicates whether the characterisation holds at all time points in a given interval or only at some of them, in the case of "in" and "throughout".

\section{Individual sentence interpretability}

The interpretability of a summary first depends on the interpretability of each sentence that composes it. Two components of this interpretability can be distinguished, discussed in turn in the following subsections: first each sentence must indeed characterise the data to be summarised, which is usually measured by a quality degree. The interpretability of a sentence is also influenced by its linguistic expression, i.e. the linguistic rendering of the characterisation whose quality has been established.

\subsection{Sentence quality}

In the protoform approach of linguistic summaries, each sentence is an instantiation of the general schema on the considered data. This section recalls the definition of the degree of quality associated with most protoforms mentioned in the previous section. It also discusses some procedures proposed to select sentences with high quality. 


\subsubsection{Degree of quality definition}

For most protoforms discussed in the previous section, the computation of the quality degree is decomposed into two steps: the quality of " $B y$ 's are $A$ " is first evaluated for each data point in the considered data set, depending on the membership functions $\mu_{A}$ and $\mu_{B}$ associated with the fuzzy modalities $A$ and $B$. The special case of " $y$ 's are $A$ " corresponds to a fuzzy set $B$ whose membership function $\mu_{B}$ is constant and equal to 1 . In the second step, these individual degrees are aggregated according to the mode $M$.

Classic protoforms. For classic protoforms " $Q B y$ 's are $A$ ", the quality is measured for each data point $x$ as

$$
v(x)=\mathrm{T}\left(\mu_{A}(x), \mu_{B}(x)\right)
$$

where $T$ denotes a t-norm. It thus measures the individual contribution of $x$ to support the considered " $B y$ 's are $A$ ", in a manner similar to the confidence definition for fuzzy association rules [21].

The aggregation of these individual contributions depends on whether $Q$ is absolute or relative: the quality degree of the summary is computed as follows in each case, respectively denoted by $q_{a}$ and $q_{r}$

$$
q_{a}=\mu_{Q}\left(\sum_{x \in \mathcal{D}} v(x)\right) \quad q_{r}=\mu_{Q}\left(\frac{\sum_{x \in \mathcal{D}} v(x)}{\sum_{x \in \mathcal{D}} \mu_{B}(x)}\right)
$$

where $\mathcal{D}$ denotes the dataset. This definition of the quality degree of protoforms is interpreted as a truth degree: it assesses the extent to which it is true that $Q$ data (or a proportion of $Q$ data) satisfy "By's are A".

The difference with the confidence of fuzzy association rules is that association rules consider interesting the cases where the obtained value is high, whereas linguistic summarisers also exploit the result when it is low, e.g. using a quantifier such as "few" in the linguistic summary.

Protoforms for time series. In the case where the data are times series, the approaches based on the extraction of numerical attributes mentioned in the previous section have the same quality measures as classic protoforms.

The approaches based on a two-step process that involves a temporal quantifier as well as a fuzzy quantifier, i.e. of the form " $Q B y$ 's are $A Q_{\tau}$ time", measure the summary quality as a twolevel aggregation [15]: each series $x=\left(x_{t}\right)_{t \in \mathcal{T}}, \mathcal{T}$ being the universe of time stamps, is individually summarised as " $Q_{\tau} B y$ 's are $A$ " using the temporal quantifier $Q_{\tau}$ applied to the data time stamps $t$; at a second level, a classic protoform lying on the quantifier $Q$ is applied to all series that make up the whole data set. Thus, each time stamp of each time series is first evaluated:

$$
v\left(x_{t}\right)=\mathrm{T}\left(\mu_{A}\left(x_{t}\right), \mu_{B}\left(x_{t}\right)\right)
$$

and the individual contribution of a time series is defined as the aggregation of $v\left(x_{t}\right)$ applying Eq. (2) to the set of dates, e.g. in the case of a relative quantifier:

$$
q_{r}=\mu_{Q}\left(\frac{\sum_{x \in \mathcal{T}} v\left(x_{t}\right)}{\sum_{x \in \mathcal{T}} \mu_{B}\left(x_{t}\right)}\right)
$$


The second step then aggregates these individual values across all considered time series, applying the same formula as in Eq. (2) again, for all $x \in \mathcal{D}$.

Temporal protoforms. In the case of temporal protoforms based on the "regularly" mode, i.e. of the form "regularly, $y$ 's are $A$ ", the quality criterion can be interpreted along the same lines as a two-step process, namely individual evaluation and aggregation, although the steps differ [17, 18]: first for each time stamp individually, a binary decision is made to decide whether it is considered as supporting $A$ or not, several methods being presented in [18]. In the second step, aggregation measures the regularity of the episodes of the two types of groups and their alternation, based on a dispersion measure of their respective durations.

In the case of fuzzy temporal propositions, quality is measured as a truth degree, whose expression depends on the persistence mode of the considered protoform, influencing both the aggregation and the individual expression $[19,20]$. Indeed, for the non-persistent mode, i.e. a summary of the form "in $M_{\tau} y$ 's are $A$ ", the validity of " $y$ 's are $A$ " at each time stamp is computed as the conjunction between the membership of the modality $A$ and the temporal reference $M_{\tau}$. The quality degree is computed as a disjunction of these values, so if one of them at least is high in the considered temporal reference then the quality degree is too:

$$
v(t)=\mathrm{T}\left(\mu_{A}\left(x_{t}\right), \mu_{M_{\tau}}(t)\right) \quad q=\perp_{t \in \mathcal{T}} v(t)
$$

where $T$ and $\perp$ respectively denote a t-norm and a t-conorm.

For the persistent mode, i.e. a summary of the form "throughout $M_{\tau} y$ 's are $A$ ", the validity of each time stamp is computed as the logical implication between $M_{\tau}$ and $A$, and the aggregation operator is a conjunction $T$, so the quality degree is high if the validity of every time stamp is high in the considered time interval:

$$
v(t)=\perp\left(\mu_{A}\left(x_{t}\right), 1-\mu_{M_{\tau}}(t)\right) \quad q=\top_{t \in \mathcal{T}} v(t)
$$

This implication expression means that the proposed method is actually an alternate computation for the summariser. It must be moreover noted that various implication operators could be used, and it would be interesting to study the different types of interpretation they would lead to.

Other quality measures. The quality measures defined above are the most usual ones for different types of generalised protoforms, which can be interpreted in the same framework.

Among other measures, the degree of appropriateness [22] compares the sentence truth value with an expected value, computed from characteristics of the data set to be summarised. Consider for instance a database containing 50\% of "young" people and 50\% of "old" people for the "Age" attribute and 50\% of "well paid" and 50\% of "badly paid" for the "Salary" attribute. If the sentence "young people are badly paid" has a truth value of 0.25 , it is not considered as interesting; however, if it is $40 \%$, then it deviates in a significant way, so its degree of appropriateness is important.

The relevance measure [23] is designed to filter out summaries about attributes not containing unusual data. It is computed as the average difference between a value and the average value for this attribute, so as a mean absolute deviation, yielding a high relevance when several individual values in the dataset are different enough from the average value. 
Informativeness, defined in [9], combines the quality degree defined as a truth value with the specificity of the quantifier and the summariser, where specificity measures the ability for a quantifier or a summariser to precisely define a single element in $\mathcal{D}$.

The differentiation score [15] aims at favouring sentences that highlight differences between subgroups of data. Its particularity is that its evaluation does not only depend on the sentence to be assessed but also on other similar sentences, requiring the definition of a similarity measure. It is then high for a sentence $S$ if the quality degree is high for $S$ and low for similar sentences. It can then lead to a new type of protoform, e.g. expressed as " $Q B_{1} y$ 's are $A$ whereas $Q B_{2} y$ 's are not", that can be illustrated in a medical application by a sentence such as "few observations related to male patients have low heart rate whereas it is not the case for female patients".

\subsection{Identification of sentences with high quality}

The number of possible summaries that can be generated from a set of quantifiers, qualifiers, summarisers and database attributes may be huge because of the number of possible combinations between these elements. So the identification of interesting phrases, i.e. sentences with a high quality, has to be performed both before and after their generation: before their generation to save computation time, and after it to save user time, i.e. to reduce the number of results he will have to read.

Various techniques are used to limit the number of summaries to evaluate, using particular properties of quantifiers or modalities to limit the number of cases to explore. For instance, when a quantifier is included in another, the largest one is explored only if the narrower one has a low truth value; if not, the evaluation with the narrower quantifier is retained and the computation with the larger quantifier is ignored [16].

Another solution consists in performing a prior evaluation in order to remove summaries whose value cannot be above a given threshold, using for instance the degree of focus proposed in [24]. This degree of focus allows the same kind of optimisation as the support in association rules [25].

Another way of limiting the number of protoforms to evaluate is the user guided summarisation, where users query the system with instances of protoforms which are evaluated and whose truth values are returned. It must be underlined that this approach does not generate all relevant sentences but checks the validity of some sentences queried by the user. FQuery [26], SummarySQL [27] or Quantirius [28] are instances of this approach.

The computation complexity with these systems depends on the level of freedom in the query. For instance, when the user asks for the summaries with a given quantifier, a given summariser and a given qualifier, only one sentence has to be evaluated. However, certain systems allow the user to be less specific, asking for instance to evaluate the sentences containing a certain summariser for all possible quantifiers, in which case the number of sentences to be computed is larger. The classification of the different queries and their complexity is given in [29].

After the generation step, various approaches have been proposed to filter the generated sentences. Thresholding methods [30] aim at focusing on interesting results only, as for instance rank-based thresholding, which selects the summaries with the top $k$ truth values, or score-based thresholding where the chosen summaries must have a truth value greater than a given threshold, as used in $[23,28]$. 
A way of automatically computing this threshold is also proposed in [30] and implemented in [28]. It relies on the evaluation of two special protoforms over the set of the $k$ summaries with the highest truth values, with $k=1 \ldots n_{s}, n_{s}$ denoting the number of returned summaries. The special protoforms are "Most of the summaries with a high truth value are selected" and "Most of the selected summaries have a high truth value". The particular $k$ returning the highest result when computing the conjunction of these protoforms is selected to return the $k$ best summaries.

In the context of times series, the evaluation of the quality degree actually performs the selection step, and produces a single sentence. This is for instance the case for the protoform " $A d v$ every $p$ units $y$ 's are $A$ " [17], where the quality is evaluated only for the most relevant $p$ values.

In addition to the quality measure, linguistic rendering also influences the interpretability of the summary, as detailed below.

\subsection{Linguistic rendering}

The interpretability of a sentence in a summary depends on its linguistic expression, both through the linguistic variables attached to the attributes and to the fuzzy quantifier.

The linguistic variables are usually defined by the user, both regarding the linguistic labels and the membership functions. In this case they can be considered as improving interpretability, since they somehow personalise the summaries to the user preference and his subjective perception of the attributes. However, such variables may be inappropriate with respect to the data structure [31]. Automatic methods to extract a partition from the data can also be considered [32].

In the case of fuzzy temporal references, several aspects of the time formulation can be considered, as proposed in [17] for the case of regularity assessment: a numerical candidate period is automatically computed from the data, then transformed into a linguistic representation emulating the way humans usually express time, with respect to time unit, time approximation, and approximation characterisation through an adverb. The choice of the unit is based on the general observation that speakers usually choose a convenient unit to express time with small numbers. As an illustration, it seems normal to declare "I meet her every week" but odd to say "I meet her every 168 hours". Once the unit is chosen, the values commonly used are often multiples of five or at least integers. For instance, one would rather say "This happens every 45 minutes" than "This happens every 43.8 minutes". It must be underlined that this appreciation is highly dependent on the context in which it is produced: it can happen that a precise time is required, e.g. for sportive events or auctions. Lastly, the adverb selection makes it possible to define the level of accuracy of statements. For a time expression, it can be an adverb as "exactly", "approximately", "nearly", "roughly", "around". For instance, instead of saying "the game lasted 1 hour and 7 minutes", one would rather say "the game lasted approximately 1 hour", adding the adverb to indicate that this period is not exact. An implementation of these principles is proposed in [17] to generate relevant linguistic expressions.

Along the same lines, the EasyText system [33] detects variations such as increase or decrease with their magnitude as a number, and reformulates it linguistically choosing the appropriate term, for instance among "double" or "triple". 


\section{Global interpretability}

The interpretability of a summary is not only based on the interpretability of each of its individual sentences but also on their interpretability as a whole. In this section, different aspects of this global interpretability are explored. First, the property of consistency of the sentences is detailed, then various methods to detect and remove redundancy are presented; finally, different techniques of information enhancement are introduced, dedicated to the creation of knowledge through links between the sentences.

It must be noted that the global interpretability presented here is related to " $Q B y$ 's are $A$ " protoforms, not to temporal protoforms.

\subsection{Consistency of the sentence set}

A summary can be considered consistent when the two properties of non-contradiction and double negation detailed below are satisfied.

Non contradiction. The non contradiction property (called "external negation" in [34] and denoted $\mathrm{NC}$ in the following) refers to the fact that two sentences with a contradictory term have complementary truth values. For instance, if $Z$ is "Most young people are tall", $Z_{1}$ is "Few young people are tall" and $Z_{2}$ is "Most young people are short", then intuitively, $Z_{1}$ and $Z_{2}$ are in contradiction with $Z$ and their truth values should be such that $q\left(Z_{1}\right)=q\left(Z_{2}\right)=1-q(Z)$.

More generally, the two contradictory forms of a protoform $P=Q B y$ 's are $A$ are defined as:

$$
C_{1}(P)=\neg Q B y \text { 's are } A \quad C_{2}(P)=Q B y \text { 's are } \neg A
$$

The negation $\neg$ can be understood in different ways as detailed below. Moreover, it must be noted that " $Q \neg B y$ 's are $A$ " is not considered as a contradiction of $P$ : it is a summary that applies to a distinct subgroup of the data, with no relation to the characteristics of the $B$ subpopulation. For instance, if the sentence $Z_{3}$ denotes "Most old people are tall", it is not in contradiction with $Z$ since there is no particular constraint on the fact that "old people" must not be as tall as "young people" are. In the case where one of the two summaries holds and not the other, a differential summary can be established (see [15] and paragraph 3.1.1).

Double negation. The double negation property (DN) (called "antonymy" in [34]) also stems from the human logical interpretation of language. It states that, when two contraries are applied to a protoform, its truth is not modified. For instance, the double negation of $Z$ is "few young people are short", is considered to have a meaning similar to "most young people are tall", if "tall" is the antonym of "short".

The double negation $D$ of a protoform $P$ is defined as:

$$
D(P)=C_{1}\left(C_{2}(P)\right)=C_{2}\left(C_{1}(P)\right)=\neg Q B y \text { 's are } \neg A
$$

so the double negation is actually a contradiction applied twice on a protoform.

The double negation property states that $q(D(P))=q(P)$. 
Consistency conditions. This paragraph states some constraints on the definition of the quantifiers and qualifiers in order to comply with the NC and DN properties.

First, the negation operator used in (7) and (8) has to be precised. In linguistic summaries, the fuzzy negation and the antonym are used. The fuzzy negation is used for both quantifiers and qualifiers, denoted $\bar{F}$, and defined as:

$$
\mu_{\bar{F}}(x)=1-\mu_{F}(x)
$$

The antonym $\hat{Q}$ applies to relative quantifiers and is defined as:

$$
\mu_{\hat{Q}}(x)=\mu_{Q}(1-x)
$$

With protoforms as " $Q y$ 's are $A$ ", the following is proven in [9]:

$$
q(Q y \text { 's are } A)=\underbrace{q(\hat{Q} y \text { 's are } \bar{A})}_{D N}=\underbrace{1-q(\bar{Q} y \text { 's are } A)}_{N C \text { with } C_{1}}
$$

meaning first that the double negation property is verified using the antonym of $Q$, i.e $\neg Q=\hat{Q}$ and the negation of $A$, i.e. $\neg A=\bar{A}$ and second that the $C_{1}$ form of the non contradiction property is validated with the negation of $Q$, i.e. $\neg Q=\bar{Q}$.

Hence, to generate consistent summaries, when a modality $A$ is specified for a summariser then its negation $\bar{A}$ has to be included as a modality as in Eq. (9), and the quantifiers must be defined pairwise, $Q$ and $\neg Q$, with $\neg Q$ verifying:

$$
1-\mu_{\neg Q}(x)=\mu_{\neg Q}(1-x)
$$

To comply with this condition, the membership function of $Q$ has to possess the point of symmetry $(0.5,0.5)$.

This constraint reduces the number of possible definitions for the quantifiers and qualifiers verifying consistency conditions as defined above, somewhat limiting their expressive power on the one hand, but easing their definition and guaranteeing interesting semantic properties on the other.

\subsection{Non redundancy}

The second aspect of summary interpretability is the absence of redundancy, the latter occurring when different sentences convey the same meaning, needlessly extending the summary. The non redundancy analysis allows to filter out useless generated sentences.

It can first be noted that the double negation presented in 4.1 is such a case of redundancy, that requires to filter out one of the two sentences $P$ or $D(P)$.

Other redundancy cases result from inclusion and similarity as discussed below. 
Inclusion. Inclusion refers to a situation where the qualifier, summariser or quantifier of a sentence is included in the qualifier, summariser or quantifier of another. For instance, "more than $80 \%$ of the young men are well paid" is included in "most young men are well paid", when the quantifier "more the $80 \%$ " has a support included in the support of "most". In the case of "Most young employees have a salary less than $\$ 5000$ " and "Most young employees have a salary about $€ 2000$ ", the latter sentence is included in the former, due to the inclusion of the summariser "about $\$ 2000$ " in "less than \$5000".

Formally, $P_{1}=Q B y$ 's are $A$ is included in $P_{2}=Q^{\prime} B y$ 's are $A^{\prime}$ if $Q \subseteq Q^{\prime}$ or $A \subseteq A^{\prime}$. Several methods of inclusion detection are described in [28].

Different decisions can be taken when an inclusion is detected. In [28], this inclusion is interpreted as a redundancy so the included sentence which is the most precise is kept whereas the other is discarded. On the contrary, in the SaintEtiQ system [35], included sentences are not removed but organised in a hierarchy, where the most general ones are located at the top of the tree, and the most specialised ones at the bottom of the hierarchy.

Similarity. Similarity between sentences can also be interpreted as redundancy. Denoting $P_{1}=$ $Q_{1} B_{1} y$ 's are $A_{1}, P_{2}=Q_{2} B_{2} y$ 's are $A_{2}, q_{1}$ and $q_{2}$ their quality degrees, the similarity can be defined as [25]:

$$
\operatorname{sim}\left(P_{1}, P_{2}\right)=\min \left(\operatorname{sim}_{1}\left(Q_{1}, Q_{2}\right), \operatorname{sim}_{1}\left(A_{1}, A_{2}\right), \operatorname{sim}_{1}\left(B_{1}, B_{2}\right), \operatorname{sim}_{2}\left(q_{1}, q_{2}\right)\right)
$$

where $\operatorname{sim}_{1}$ computes a similarity between two fuzzy sets and $\operatorname{sim}_{2}$ a similarity between two real values.

It advantageously takes into account all components of a summary, namely its quantifier, qualifier, summariser and truth value.

When two sentences in the set of generated phrases are too similar, depending for instance on a threshold defined by the user, one can be removed. It must be noted that this similarity measure can also encompass the definition of inclusion, depending on the definition of the $\operatorname{sim}_{1}$ and $\operatorname{sim}_{2}$ functions.

\subsection{Information}

The third point studied about summary interpretability is the knowledge it brings to the user, not as the sum of the information conveyed by each of its individual sentences, but rather through the links existing between them.

In this subsection, several sources of information based on the relations between sentences are presented: sentence inference and underlying meaning.

\subsubsection{Sentence inference}

Causality between sentences allows to enhance their meaning, either by reasoning with them or by highlighting their link.

For instance, from the two summaries " $Q_{1} A y$ 's are $B$ " and " $Q_{2} B y$ 's are $C$ ", a new piece of knowledge of the form " $Q y$ 's are $A$ and $C$ " where $Q$ is e.g. defined as the multiplication of the fuzzy numbers $Q_{1}$ and $Q_{2}$ [36]. More developments on the combination of summaries have been 
proposed in works on syllogistic reasoning $[12,37]$. Nonetheless, this kind of reasoning is useful only if $B \subset A$, otherwise the resulting quantifier $Q$ is the vacuous quantifier none - to - all ([38]).

In Natural Language Generation, causality can be expressed in words in the summary so as to improve its meaning. For instance, the BT-45 system [39] returns "Fi02 increased so saturation rose" if a causality is identified between "increase $\mathrm{FiO} 2$ " and "saturation rise".

\subsubsection{Underlying meaning}

Underlying meaning consists in the automatic evaluation of sentences whose computation seems relevant given the results obtained by means of other sentences.

For instance a sentence like "Most big books do not sell well" would influence a publisher to ask his authors to write small books. But small books may not sell well either, which he would have figured out if he had checked the truth value of "Most books do not sell well".

More generally, all $B$ modalities in " $Q B y$ 's are $A$ " must be explored in order to discover the underlying meanings. When all the sentences based on the modalities of $B$ have a high quality degree, then all " $Q B y$ 's are $A$ " sentences can be replaced by a single " $Q y$ 's are $A$ ". This equivalence can also be stressed with the protoform " $Q y$ 's are $A$ irrespective of $\operatorname{attr}(B)$ ", where $\operatorname{attr}(B)$ is the attribute of the modality $B$, e.g. the attribute "size" for the modalities "big" and "small". An instance of this protoform is "Most books do not sell well, irrespective of their size".

On the contrary, when two distinct modalities return a different quality degree, a differential summary [15] can be used, for instance "Most big books do not sell well, unlike the small ones".

The underlying meaning can be viewed as the semantic adaptation of some reduction techniques used for fuzzy rules.

\section{Conclusion and future works}

This paper is dedicated to a general form of fuzzy linguistic summaries for numerical data, based on so-called generalised fuzzy protoforms, handling specific types of data such as temporal ones. We have focused on their interpretability, which has not been much studied, unlike the one of fuzzy rule bases. We have investigated an approach of their interpretability different from the approach used for fuzzy rule-based systems, even though they clearly have some aspects in common. Further work will have to bring back together both approaches in a global view of the interpretability of fuzzy models.

Considering a summary as a set of fuzzy generalised protoforms, we have proposed to control the interpretability at both levels of individual protoforms and global summary. The generality of these protoforms makes it difficult to consider all situations and this paper represents a first attempt to evaluate the interpretability of fuzzy linguistic summaries and to propose methods to increase it. Further works on fuzzy quantifiers and their properties will complete this approach to help to consider types of protoforms which have yet not been studied, in particular from the global interpretability point of view.

\section{References}

[1] J. Casillas, O. Cordón, F. Herrera, L. Magdalena, Interpretability improvements to find the balance interpretability-accuracy in fuzzy modeling: an overview, in: Interpretability Issues in Fuzzy Modeling, 2003, pp. 3-22. 
[2] M. Gacto, R. Alcalá, F. Herrera, Interpretability of linguistic fuzzy rule-based systems: An overview of interpretability measures, Information Sciences 181 (20) (2011) 4340-4360.

[3] S.-M. Zhou, J. Gan, Low-level interpretability and high-level interpretability: a unified view of data-driven interpretable fuzzy system modelling, Fuzzy Sets and Systems 159 (23) (2008) 3091-3131.

[4] J. Casillas, Interpretability Improvements to Find the Balance Interpretability-Accuracy in Fuzzy Modeling: An Overview, in: Interpretability Issues in Fuzzy Modeling, Vol. 128, 2003, pp. 3-22.

[5] J. Yu, E. Reiter, J. Hunter, S. Sripada, SumTime-Turbine: A Knowledge-Based System to Communicate Gas Turbine Time-Series Data, in: Developments in Applied Artificial Intelligence, 2003, pp. 379-384.

[6] A. Law, Y. Freer, J. Hunter, R. Logie, N. McIntosh, J. Quinn, A comparison of graphical and textual presentations of time series data to support medical decision making in the neonatal intensive care unit., Journal of clinical monitoring and computing 19 (3) (2005) 183-94.

[7] L. Arguelles, G. Triviño, I-struve: Automatic linguistic descriptions of visual double stars, Engineering Applications of Artificial Intelligence 26 (9) (2013) 2083-2092.

[8] J. Kacprzyk, S. Zadrozny, Comprehensiveness and interpretability of linguistic data summaries: A natural language focused perspective, in: Proc. of IEEE SSCI CIHLI'13, 2013, pp. 33-40.

[9] R. Yager, A new approach to the summarization of data, Information Sciences 28 (1) (1982) 69-86.

[10] J. Kacprzyk, R. Yager, Linguistic summaries of data using fuzzy logic, Int. Journal of General Systems 30 (2) (2001) 133-154.

[11] J. Kacprzyk, S. Zadrozny, Protoforms of Linguistic Data Summaries: Towards More General Natural-LanguageBased Data Mining Tools, in: Soft computing systems, 2002, pp. 417-425.

[12] L. Zadeh, A computational approach to fuzzy quantifiers in natural languages, Computers \& Mathematics with Applications 9 (1) (1983) 149-184.

[13] J. Kacprzyk, R. Yager, "Softer" optimization and control models via fuzzy linguistic quantifiers, Information Sciences 34 (2) (1984) 157-178.

[14] J. Kacprzyk, A. Wilbik, S. Zadrozny, Linguistic summarization of time series using a fuzzy quantifier driven aggregation, Fuzzy Sets and Systems 159 (12) (2008) 1485-1499.

[15] R. J. Almeida, M.-J. Lesot, B. Bouchon-Meunier, U. Kaymak, G. Moyse, Linguistic Summaries of Categorical Time Series Patient Data, in: Proc. of FUZZ-IEEE'13, 2013, pp. 1-8.

[16] R. Castillo-Ortega, N. Marín, D. Sánchez, Linguistic local change comparison of time series, in: Proc. of FUZZIEEE'11, 2011, pp. 2909-2915.

[17] G. Moyse, M.-J. Lesot, B. Bouchon-Meunier, Linguistic summaries for periodicity detection based on mathematical morphology, in: Proc. of IEEE SSCI FOCI'13, 2013, pp. 106-113.

[18] G. Moyse, M.-J. Lesot, B. Bouchon-Meunier, Mathematical morphology tools to evaluate periodic linguistic summaries, in: Proc. of FQAS'13, 2013, pp. 257-268.

[19] P. Cariñena, A. Bugarín, M. Mucientes, S. Barro, A language for expressing expert knowledge using fuzzy temporal rules, in: Proc. of EUSFLAT-ESTYLF Joint Conf. 1999, 1999, pp. 171-174.

[20] P. Cariñena, A. Bugarín, M. Mucientes, S. Barro, A language for expressing fuzzy temporal rules, Mathware \& soft computing 7 (2) (2000) 213-227.

[21] D. Dubois, E. Hüllermeier, H. Prade, A note on quality measures for fuzzy association rules, in: Proc. of IFSA, 2003, pp. 346-353.

[22] J. Kacprzyk, S. Zadrozny, Linguistic database summaries and their protoforms: towards natural language based knowledge discovery tools, Information Sciences 173 (4) (2005) 281-304.

[23] A. Van der Heide, G. Triviño, Automatically Generated Linguistic Summaries of Energy Consumption Data, in: Proc. of ISDA'09, 2009, pp. 553-559.

[24] J. Kacprzyk, A. Wilbik, Towards an efficient generation of linguistic summaries of time series using a degree of focus, in: Proc. of NAFIPS'09, 2009, pp. 1-6.

[25] A. Wilbik, J. M. Keller, A distance metric for a space of linguistic summaries, Fuzzy Sets and Systems 208 (2012) 79-94.

[26] J. Kacprzyk, S. Zadrozny, Fuzzy querying for Microsoft Access, in: Proc. of the IEEE Conf. on Fuzzy Systems, 1994, pp. 167-171.

[27] D. Rasmussen, R. Yager, Summary SQL - A fuzzy tool for data mining, Intelligent Data Analysis 1 (1-4) (1997) 
49-58.

[28] D. Pilarski, Linguistic Summarization of Databases with Quantirius: a Reduction Algorithm for Generated Summaries, Int. Journal of Uncertainty, Fuzziness and Knowledge-Based Systems 18 (3) (2010) 305-331.

[29] J. Kacprzyk, S. Zadrozny, Fuzzy Linguistic Data Summaries as a Human Consistent, User Adaptable Solution to Data Mining, in: Do Smart Adaptive Systems Exist?, Vol. 173, 2005, pp. 321-340.

[30] S. Zadrozny, J. Kacprzyk, Computing with words for text processing: An approach to the text categorization, Information Sciences 176 (4) (2006) 415-437.

[31] M.-J. Lesot, G. Smits, O. Pivert, Adequacy of a User-Defined Vocabulary to the Data Structure, in: Proc. of FUZZ-IEEE'13, 2013, pp. 1-8.

[32] S. Guillaume, B. Charnomordic, Generating an Interpretable Family of Fuzzy Partitions From Data, IEEE Trans. on Fuzzy Systems 12 (3) (2004) 324-335.

[33] L. Danlos, F. Meunier, V. Combet, EasyText: an Operational NLG System, in: Proc. of ENLG'11, 2011, pp. 139-144.

[34] S. Barro, A. Bugarín, P. Cariñena, F. Díaz-Hermida, A framework for fuzzy quantification models analysis, IEEE Trans. on Fuzzy Systems 11 (1) (2003) 89-99.

[35] G. Raschia, N. Mouaddib, A fuzzy-based heuristic measure evaluating quality of a concept partition: application to SaintEtiQ, a database summarization system, in: Proc. of FUZZ-IEEE'00, Vol. 2, 2000, pp. 957-960.

[36] R. Yager, Connectives and quantifiers in fuzzy sets, Fuzzy Sets and Systems 40 (1) (1991) 39-75.

[37] L. Zadeh, Syllogistic reasoning as a basis for combination of evidence in expert systems, Proc. of IJCAI'85 1 (1985) 417-419.

[38] L. Zadeh, Syllogistic reasoning in fuzzy logic and its application to usuality and reasoning with dispositions, IEEE Trans. on Systems, Man, and Cybernetics SMC-15 (6) (1985) 754-763.

[39] F. Portet, E. Reiter, J. Hunter, S. Sripada, Automatic generation of textual summaries from neonatal intensive care data, in: Artificial Intelligence in Medicine, 2007, pp. 227-236. 